\title{
Role of Ultrasound in the Evaluation of Abnormal Vaginal Bleeding in Nonpregnant Patients in Reproductive Age
}

\author{
Bailey Wilson, Sanja Kupesic Plavsic
}

\begin{abstract}
Abnormal uterine bleeding is defined as any alteration in the volume, pattern or duration of menstrual blood flow. Abnormal uterine bleeding can be due to a number of organic and dysfunctional causes. This article presents different case scenarios of nonpregnant reproductive age patients presenting with abnormal genital tract bleeding. These cases will allow the reader to identify differential diagnoses related to each scenario and to understand ultrasound findings typical for nonpregnant patients presenting with abnormal vaginal bleeding. The role of B-mode and color Doppler ultrasound in the evaluation of abnormal uterine bleeding is described. Standard treatment methods are also listed for each case.
\end{abstract}

Keywords: Menorrhagia, Anovulatory cycle, Ovulatory cycle, B-mode ultrasound, Color Doppler ultrasound.

How to cite this article: Wilson B, Plavsic SK. Role of Ultrasound in the Evaluation of Abnormal Vaginal Bleeding in Nonpregnant Patients in Reproductive Age. Donald School J Ultrasound Obstet Gynecol 2012;6(1):112-120.

Source of support: Nil

Conflict of interest: None declared

\section{INTRODUCTION}

Abnormal genital tract bleeding can involve heavy or prolonged periods, frequent periods, intermenstrual bleeding, light periods, infrequent periods or the complete absence of periods. ${ }^{1}$ It is estimated that the prevalence of abnormal vaginal bleeding among women of reproductive age is as high as $30 \% .^{2}$ Being the single most common reason for gynecological referral in the United States, it is estimated that the annual direct and indirect economic costs of abnormal uterine bleeding in the United States in 2007 were approximately $\$ 1$ billion and $\$ 12$ billion respectively. ${ }^{1,2}$ Women with heavy periods work an estimated 3.6 fewer weeks per year and lose an estimated $\$ 1,692$ annually in wages compared with other women in the general workforce. ${ }^{3}$ It is, thus, very important to recognize the underlying causes and mechanisms for abnormal genital tract bleeding and to assess and manage care appropriately.

The female menstrual cycle consists of cyclic uterine bleeding that occurs approximately every 28 days, with a normal range of 21 to 35 days. Normal uterine bleeding lasts 3 to 5 days with about 30 to $50 \mathrm{ml}$ of blood loss per cycle. Menorrhagia is the term used to describe heavy or prolonged periods. Metrorrhagia is the term used to describe intermenstrual bleeding. Menometrorrhagia is excessive or prolonged bleeding at irregular intervals. Polymenorrhea describes periods that occur more frequently than every 21 days, while oligomenorrhea describes periods that occur every 35 days or more. ${ }^{4}$

The menstrual cycle results from the choreographed actions of the hypothalamus, pituitary gland, ovaries and the uterus and its endometrial lining. Alterations in any of these factors can result in abnormal uterine bleeding. Causes of abnormal genital tract bleeding can include neoplastic growth within the uterus, hormonal dysfunction, infection, coagulopathy, endometrial abnormality and ovarian dysfunction. In this article we will review the principles of ultrasound diagnosis of abnormal genital tract bleeding in reproductive age and apply it to the presented case studies. After completion of this case-based discussion the learners will be able to identify the differential diagnosis for reproductive age abnormal genital tract bleeding, list the ultrasound findings typical for patients presenting with anovulation, polycystic ovarian syndrome, functional cyst, breakthrough bleeding, submucosal fibroid, endometrial polyp and adenomyosis, and to identify the role of B-mode and color Doppler ultrasound in the diagnosis and treatment of these clinical entities.

\section{CASE STUDIES}

\section{Anovulation}

A 23-year-old nulliparous obese female presents complaining of irregular menstrual periods since menarche which began at age 12. Her last menstrual period was 4 months ago. She also states that she suffers from severe acne, which began at about the same time as her first period. Overtime, she has also noticed a slow growth of hair on her face, chin and abdomen.

She denies changes in her voice, changes in vision, headaches or nipple discharge. Her medical history is noncontributory. She states that she is not taking any medication and is not sexually active.

On physical examination, she is markedly obese. Her BMI measures 31. She has visible facial hair and abdominal hair. On pelvic examination, no adnexal masses are noted.

Laboratory studies revealed negative beta-hCG, normal TSH, normal prolactin and normal lipid profiles. Her FSH on day of 3 of the menstrual cycle was normal; however, her LH was elevated. Her free testosterone was also elevated. 


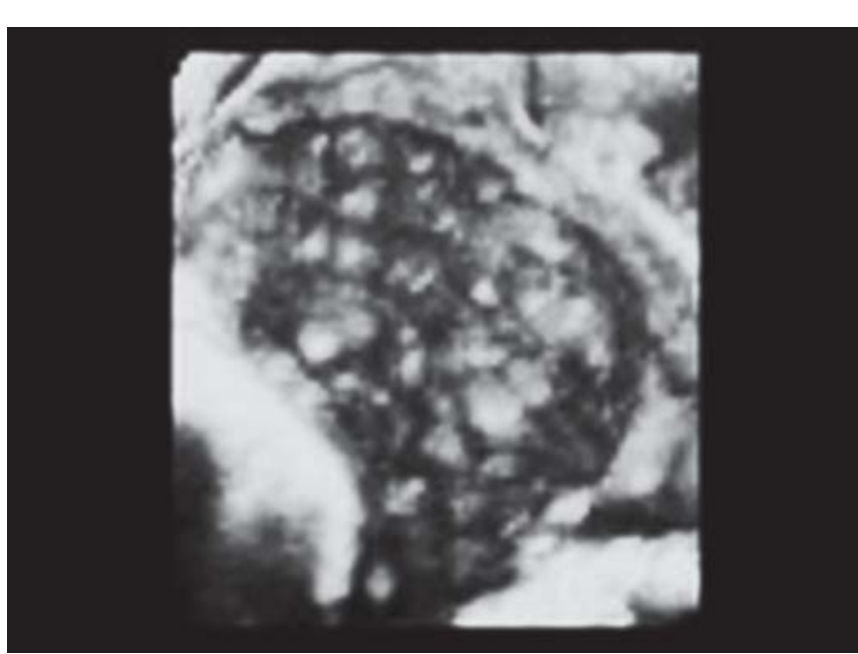

Fig. 1A: Three-dimensional ultrasound/surface rendering of an ovary in a 23-year-old patient presenting with oligomenorrhea and hirsuitism. Note: More than 10 follicles at the periphery of enlarged ovarian stroma

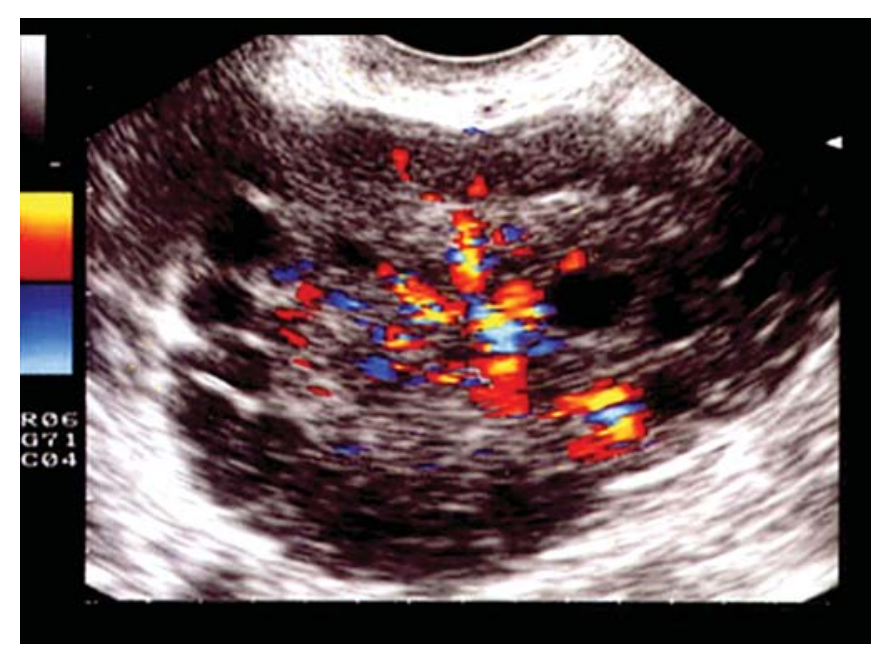

Fig. 1B: Transvaginal color Doppler ultrasound of the same patient. Note: Dilated intraovarian vessels

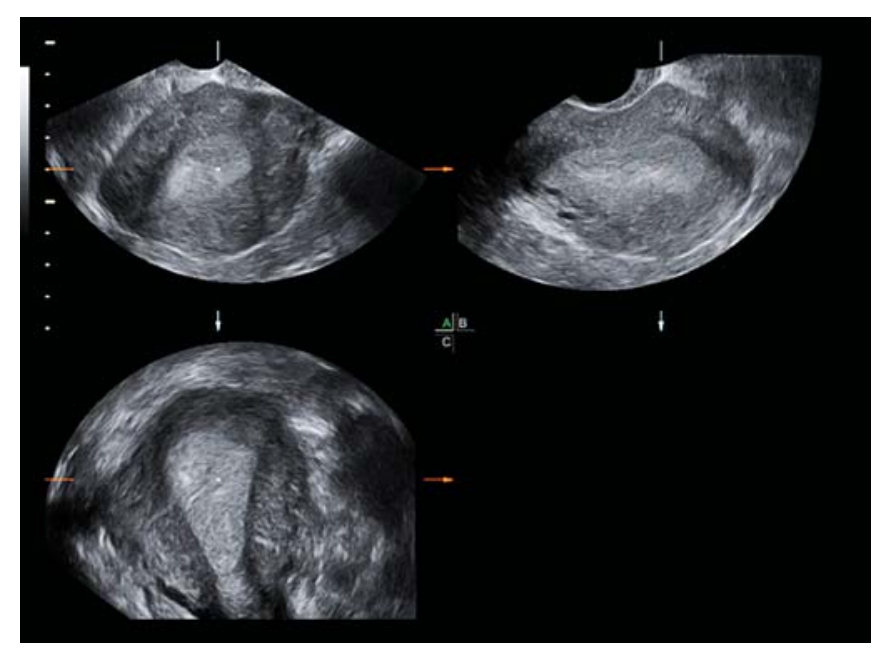

Fig. 1C: Three-dimensional image of diffusely thickened and hyperechogenic endometrium in the same patient presenting with abnormal uterine bleeding

Pelvic ultrasound is performed and reveals polycystic ovaries with a 'string-of-pearls' appearance and a uterus with a thickened endometrium (Figs $1 \mathrm{~A}$ to $\mathrm{C}$ ). She is given medroxyprogesterone acetate and she reports that menstruation began 4 days later.

A diagnosis of polycystic ovarian syndrome is made. She is advised to control her weight with diet and exercise. She is also given oral contraceptive pills to promote cyclic, predictable, menstrual periods. She is advised that if she wants to become pregnant at some point, she will need to stop the oral contraceptive pills and begin treatment with clomiphene citrate to induce ovulation. ${ }^{5}$

\section{Cervicitis and Pelvic Inflammatory Disease (PID)}

A 26-year-old nulliparous woman presents complaining of a 6-week history of thick, yellow vaginal discharge as well as vaginal bleeding after intercourse. She also states that intercourse is sometimes painful. She has multiple sexual partners and has been sexually active since age 14 . She does not use any contraceptive methods. Her last menstrual period was 2 weeks ago and was normal.

She denies fever, headaches, nausea, vomiting, abdominal pain, pelvic pain or dysuria. Her medical history is unremarkable. She is not currently on any medications.

On physical examination, she does not appear to be in any acute distress, and her physical examination findings are within normal limits. However, speculum examination reveals a mucopurulent yellow discharge. Upon bimanual pelvic examination, she exhibits cervical motion tenderness and bilateral adnexal fullness.

Laboratory studies revealed negative beta-hCG. Cervical cultures and DNA probes revealed coinfection with Neisseria gonorrhea and Chlamydia trachomatis.

Transvaginal B-mode and color Doppler ultrasound demonstrate dilated and fluid filled tubes (Figs 2A and B).

She is given $1 \mathrm{gm}$ azithromycin to treat both the Neisseria and the chlamydial infections. Her current partner is also treated and she is counseled on safe sex practices and condom use. ${ }^{6}$

\section{Submucosal Fibroid}

A 42-year-old G4P3013 female complains of menorrhagia and dysmenorrhea for the past 6 months of her menstrual periods. Her periods occur every 28 days and used to last for 5 days, but are now lasting up to 10 to 14 days at a time. She states that she feels constantly tired and has episodes of dizziness and headaches throughout the days that she bleeds. She takes ibuprofen for the headaches and the dysmenorrhea without relief.

She denies shortness of breath, chest palpitations and blurry vision. She also denies nausea, vomiting, abdominal pain, diarrhea and constipation. 


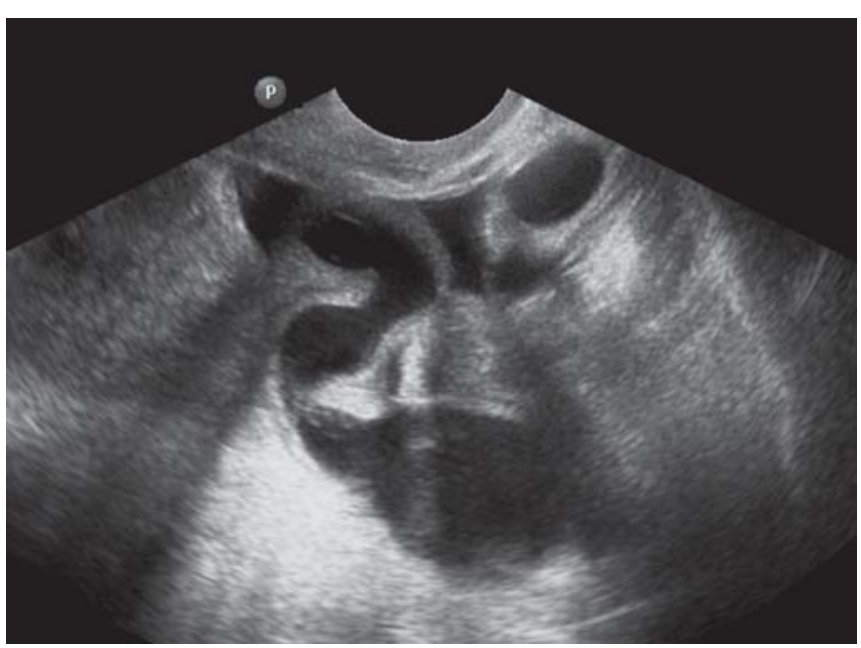

Fig. 2A: Transvaginal ultrasound of dilated and fluid filled tube in a patient presenting with vaginal discharge and postcoital bleeding

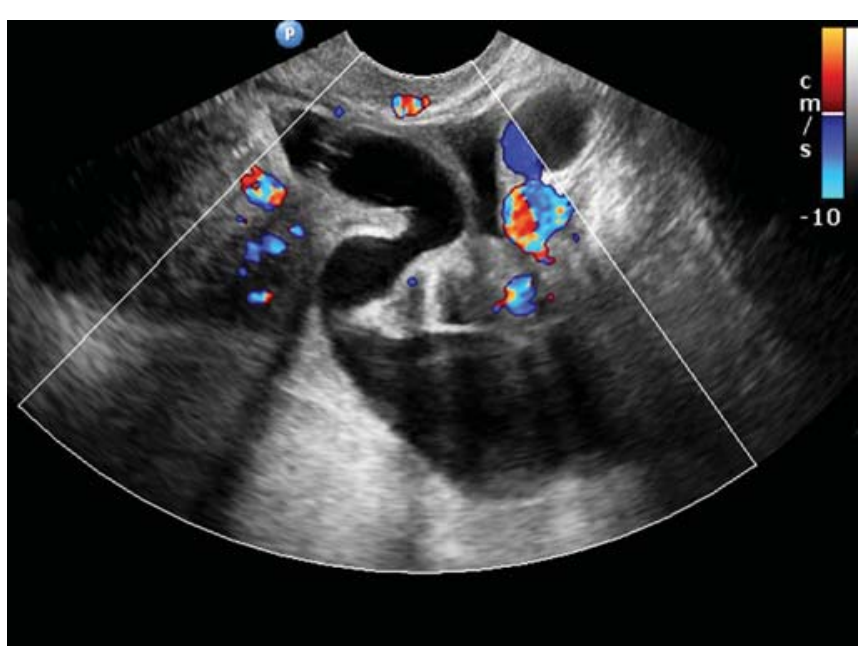

Fig. 2B: The same patient assessed by color Doppler ultrasound Note: Increased vascularization at the periphery of hydrosalpinx. Color Doppler clearly differentiates hydrosalpinx from pelvic varicosities

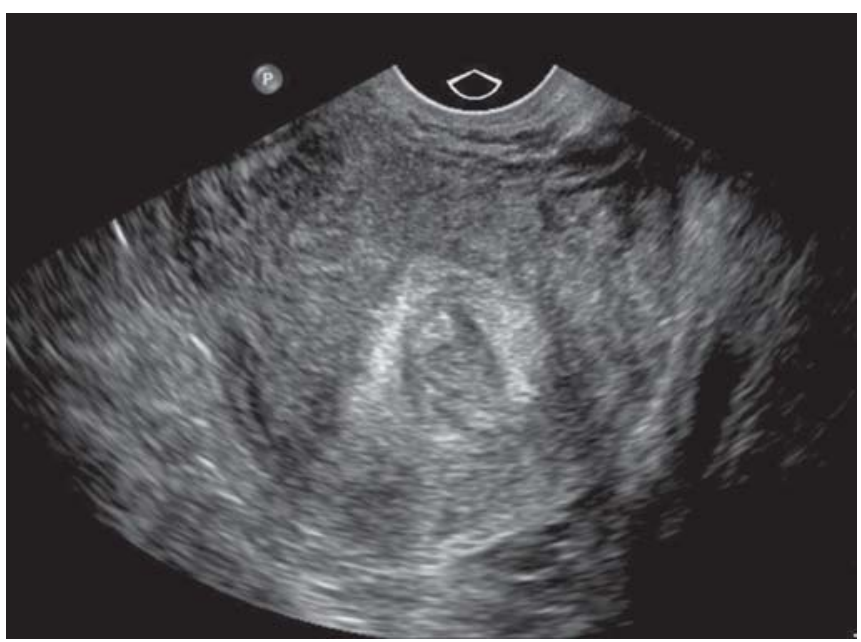

Fig. 3: Transvaginal ultrasound of a submucosal uterine fibroid surrounded by hyperechogenic endometrium

On physical examination, she is visibly pale. Her blood pressure is $140 / 90$, heart rate is 85 beats per minute, respiratory rate is 20 breaths per minute and temperature is $98.6^{\circ} \mathrm{F}$. Her lungs are clear to auscultation bilaterally. Her abdomen is soft and nontender. On pelvic examination, the uterus is enlarged and no adnexal masses are palpated.

Laboratory studies revealed negative beta-hCG. Her hemoglobin level is $9.5 \mathrm{gm} / \mathrm{dl}$ and her hematocrit is $25 \%$.

Transvaginal ultrasound showed a hypoechoic fibroid measuring $4 \times 3 \mathrm{~cm}$, originating from the inner layer of the myometrium (Fig. 3). Saline-infusion sonography revealed similar findings. Endometrial biopsy revealed secretory transformed endometrium.

A diagnosis of uterine submucosal leiomyoma is made. She is given a GnRH agonist to attempt to shrink the leiomyoma prior to therapeutic hysteroscopy.

\section{von Willebrand's Disease}

A 15-year-old nulliparous female complains of menorrhagia, headaches, dizziness, shortness of breath and chest palpitations. She goes through a tampon every hour when she is menstruating and she feels so weak during her periods that she has to miss her high school classes. She says that these perimenstrual symptoms have been going on for quite a while, but that she feels these to be getting worse lately. Menarche began at age 13 and the patient states that she remembers her period to be consistently heavy since it started. She also states that she gets frequent nosebleeds and she bruises very easily. Her mother and maternal aunt suffer from similar menstrual symptoms.

On physical examination, the patient has remarkable pallor and she looks weak and fatigued. Her blood pressure is $90 / 50$, heart rate is 90 beats/minute, respiratory rate is 22 breaths per minute and temperature is $98.6^{\circ} \mathrm{F}$. Examination of her oropharynx reveals noticeable gum bleeding. Her lungs are clear to auscultation bilaterally. Her heart sounds are tachycardic, but no murmurs or gallops are noted. Her abdomen is soft and nontender with normal bowel sounds auscultated. Her capillary refill time is greater than 3 seconds. Upon examination of her extremities, multiple purpuric regions are noticed. Pelvic examination revealed no abnormalities.

Laboratory examination revealed negative beta-hCG. Her hemoglobin level is $6.8 \mathrm{gm} / \mathrm{dl}$ and her hematocrit is $20 \%$. Her bleeding time and partial thromboplastin time are elevated, while her platelet count and prothrombin time are within normal limits. von Willebrand's factor (vWF) levels are decreased. Ristocetin cofactor assay revealed abnormal agglutination.

Pelvic ultrasound reveals no abnormalities (the uterus and the ovaries were normal size, with no evidence of uterine and/or adnexal masses).

A diagnosis of von Willebrand's disease is made. Her decreased levels of vWF create a defect in platelet plug 
formation, causing an increase in her bleeding time. The decrease in vWF also creates a coagulation defect since, vWF acts to carry and protect factor VIII, part of the intrinsic coagulation cascade. Thus, her partial thromboplastin time is elevated. Since, a decrease in vWF has no effect on platelet structure or function, her platelet count is normal. In addition, vWF plays no role in the extrinsic coagulation cascade and thus, her prothrombin time is normal. The ristocetin cofactor assay is a test used to diagnose and monitor von Willebrand's disease. This patient's test revealed an abnormal agglutination response upon administration of the ristocetin cofactor assay, confirming the diagnosis of von Willebrand's disease. ${ }^{7}$

The patient is suffering from severe symptomatic anemia and is admitted to the hospital for blood transfusion. Upon discharge, she is given desmopressin acetate (DDAVP) to help raise vWF levels in her body.

\section{Adenomyosis}

A 38-year-old multiparous female presents with menorrhagia and painful menses for the past year. She states that she begins to feel cramp-like pain beginning about 1 week before the onset of menses and lasting through the final day of menstrual flow. She says she has to change her pad every hour when she is menstruating.

She denies headaches, change in vision, chest pain, difficulty breathing, nausea, vomiting, dizziness or abdominal pain.

Her physical examination is within normal limits. On pelvic examination, her cervix is noted to be normal. Upon palpation, she has a diffusely enlarged globular uterus. No adnexal masses or tenderness are noted.

Laboratory studies revealed negative beta-hCG. Her hemoglobin is $10.0 \mathrm{gm} / \mathrm{dl}$ and her hematocrit is $29 \%$.

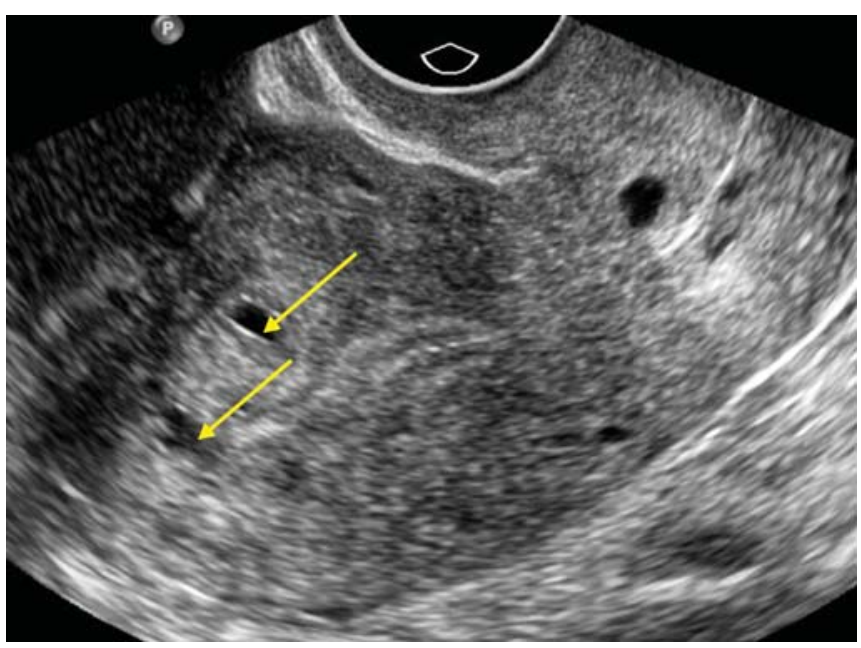

Fig. 4: Enlarged uterus with heterogeneous myometrium. Note: Irregular junctional zone and a few cystic lesions within the myometrium in a patient with severe adenomyosis
Pelvic ultrasound imaging showed a heterogenous myometrium with streaky lines with posterior shadowing and multiple cystic structures within the myometrium (Fig. 4).

The patient is given oral contraceptive pills to manage her menstruation and she is advised that the only definitive treatment for adenomyosis is hysterectomy.

\section{Functional Cyst and Dysfunctional Bleeding}

A 22-year-old nulliparous female presents with complaints of irregular menstrual bleeding for the past 2 months. She states that her periods have always been regular since menarche at age 12 but for the past 2 months, her cycles have been shorter. Menstruation started 1 week prior to the normal onset of flow.

She denies any vaginal discharge and pelvic pain. She also denies other physical complaints.

On physical examination, she is afebrile and in no acute distress. On pelvic examination, an enlarged right-sided ovary is palpated. She notes tenderness to palpation of the right ovary. Her left ovary is unremarkable. Her vagina, cervix and uterus are within normal limits.

Laboratory studies revealed negative beta-HCG. Gonorrhea and chlamydial cultures were also negative.

A pelvic ultrasound is ordered that reveals a $4 \times 6 \mathrm{~cm}$ follicular cyst of the right ovary (Fig. 5) and a thickened endometrium. Color Doppler depicts periovarian vessels, which virtually excludes adnexal torsion.

A diagnosis of a functional follicular cyst is made. The patient is advised to return for a repeat pelvic ultrasound in 6 weeks, as these follicular cysts usually resolve on their own. The patient is also advised that if the cyst persists, she may need to begin combination oral contraceptive therapy to control ovulation.

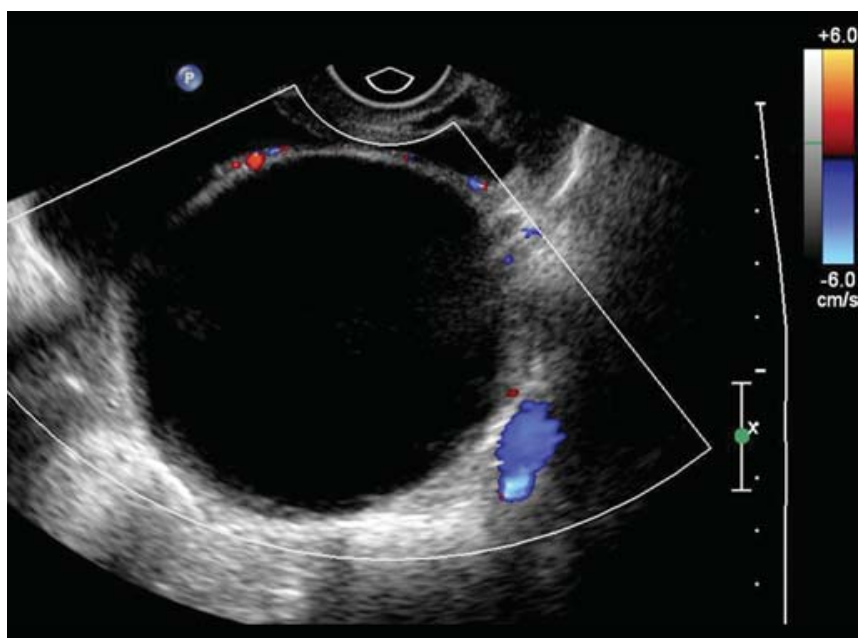

Fig. 5: Transvaginal color Doppler scan of a unilocular cyst. Note: The thin walls, clear fluid content and pericystic blood flow signals 


\section{Endometrial Polyp}

A 33-year-old multiparous female presents with symptoms of intermenstrual vaginal spotting and heavy and prolonged menstrual periods for the past 3 months. She states that her periods have always been regular with normal flow, but lately they have become worse. She says that on her bleeding days, she will go through a pad every 2 hours. She will typically bleed for 2 to 3 days longer than she used to. She also complains of headaches and shortness of breath upon minimal exertion.

On physical examination, she looks pale but does not appear to be in any acute distress. Her blood pressure is $100 / 60$ and her heart rate is 90 beats/minute. Her respiratory rate is 20 breaths/minute. Her temperature is $97.9^{\circ} \mathrm{F}$. Her conjunctivae are pale but no other abnormalities in the eyes are noted. Her lungs are clear to auscultation bilaterally. Her cardiac examination revealed no abnormalities. Her abdomen is soft and nontender. Bowel sounds are present. Her capillary refill time is 3 seconds. Pelvic examination showed no abnormalities.

Laboratory studies revealed negative beta-hCG. Gonorrhea and chlamydial cultures are also negative. Her hemoglobin is $10.0 \mathrm{gm} / \mathrm{dl}$ and her hematocrit is $29 \%$.

Saline infusion sonography reveals a hyperechogenic elongated focal lesion protruding into the uterine cavity. ${ }^{8}$ Transvaginal ultrasound showed a hyperechogenic endometrium with focal mass within the intrauterine cavity. Saline infusion sonography showed two echogenic lesions, outlined by fluid (Fig. 6).

A diagnosis of endometrial polyps is made. The patient is informed of the diagnosis and therapeutic hysteroscopy is scheduled for removal of the polyp.

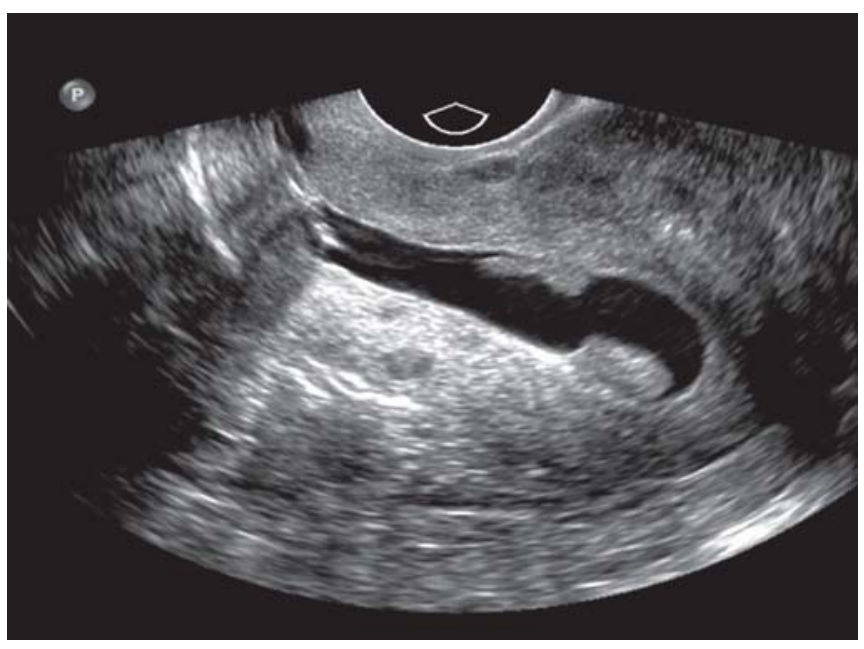

Fig. 6: Saline infusion sonography (SIS) outlines two endometrial polyps

\section{Hypothyroidism}

A 28-year-old nulliparous female presents complaining of menorrhagia for the past 6 months. She states her periods are heavier and more prolonged than before. She also complains of recent episodes of constipation, headaches and a generally depressed mood. She attributes this depression to her recent weight gain of 20 pounds and the inability to conceive with her husband.

She denies any changes in vision, chest palpitations, shortness of breath or abdominal pain.

On physical examination, the patient looks mildly overweight and has slight facial edema. However, the patient is not in any acute distress. Her blood pressure is $100 / 60$, temperature is $97.1^{\circ} \mathrm{F}$, respiratory rate is 18 breaths/minute and heart rate is 55 beats/minute. Her thyroid is enlarged on palpation. Her lungs are clear to auscultation bilaterally. Her cardiac examination revealed regular rhythm with mild bradycardia. No murmurs or gallops were auscultated. Her abdomen is soft and nontender. Bowel sounds are present. Her extremities showed no edema and her deep tendon reflexes were decreased bilaterally. Pelvic examination revealed no abnormalities.

Her laboratory studies revealed negative beta-hCG. Her hemoglobin and hematocrit were normal. TSH was elevated. T3 and T4 were decreased. Antithyroid peroxidase test (anti TPO test) is positive, indicating autoimmune Hashimoto's thyroiditis. Prolactin levels were normal. Transvaginal ultrasound reveals normal uterine and ovarian findings.

Thyroid ultrasound detects enlarged and heterogeneous thyroid gland (Fig. 7). Laboratory and ultrasound findings are diagnostic of hypothyroidism, and the patient is started on levothyroxine.

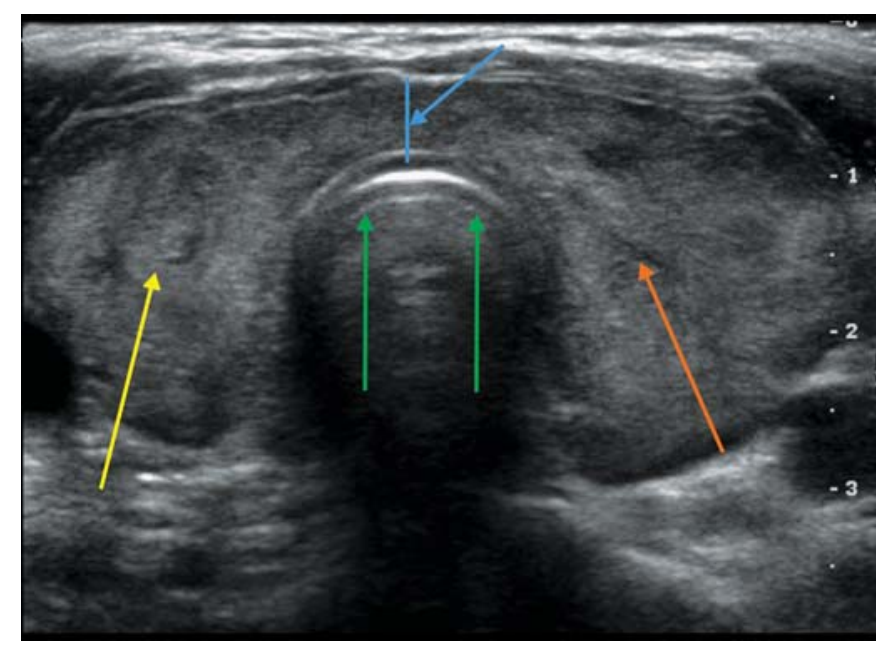

Fig. 7: Neck ultrasound/transverse image showing enlarged thyroid gland. Note: Right lobe of a thyroid (yellow arrow), left lobe of a thyroid (orange arrow), isthmus (blue line and blue arrow), trachea (green arrows) and tracheal cartilage (red arrow) 
She is advised that once her body has time to adjust to this new hormone, her periods should start to become more regular and the heavy bleeding should subside.

\section{Hyperthyroidism}

A 31-year-old female presents complaining of amenorrhea for the past 4 months. Three months before that she noticed that her periods were becoming lighter and were shorter in duration. She states that her periods always used to be regular and of normal flow since menarche at age 12. She also complains of chest palpitations and diarrhea for the past several months. She has noticed that she has lost 12 pounds in the last 3 months, despite having a normal appetite and with no change in her eating or exercise habits.

She denies headaches, shortness of breath, nausea, vomiting or constipation.

On physical examination, the patient has visible proptosis and eye muscle weakness. She is also mildly diaphoretic. Her blood pressure is $130 / 85$. Her temperature is $98.6^{\circ} \mathrm{F}$. Her respiratory rate is 19 breaths/min. Her heart rate is 95 beats/minute. Her thyroid gland is enlarged and tender. Her chest is clear to auscultation bilaterally. Her abdomen is soft and nontender. Bowel sounds are present. Her extremities are nonedematous, but her deep tendon reflexes are increased bilaterally. Her pelvic examination and transvaginal ultrasound examination are normal.

Laboratory studies reveal negative beta-HCG. Her hemoglobin and hematocrit levels are within the normal limits. TSH is decreased and her T3 and T4 are elevated. Prolactin levels are normal.

Thyroid ultrasound reveals hypoechoic coarse echotexture of the thyroid gland with clear evidence of a well delineated nodule (Fig. 8A). Color Doppler depicts increased peripheral flow (Fig. 8B).

A diagnosis of hyperthyroidism is made and the patient is scheduled for fine needle aspiration biopsy or FNAB.

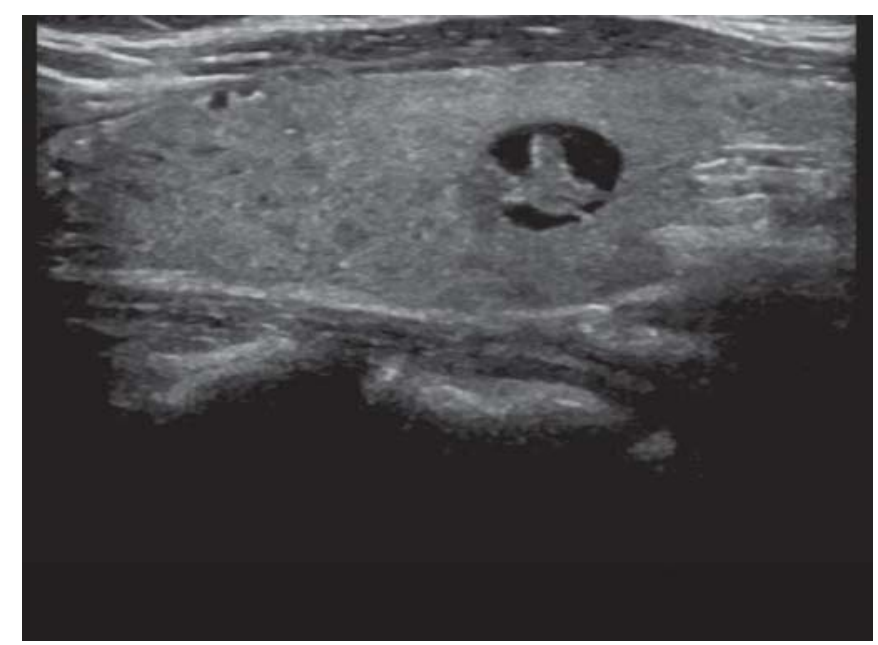

Fig. 8A: Neck ultrasound showing enlarged thyroid gland in a patient with hyperthyroidism. The parenchyma is diffusely heterogeneous and a solitary nodule is clearly identified
Under local anesthesia, a thick needle is used to obtain a 'core' tissue sample of analysis. Thyroid adenoma was confirmed by histology, and the patient was started on beta blockers to treat her symptoms and propylthiouracil to treat the hyperthyroidism. She is advised that radioactive iodine ablation or surgery may need to be considered, if this treatment does not resolve her symptoms within 1 year. She is further advised that if she becomes pregnant, radioactive iodine ablation would be contraindicated.

\section{DISCUSSION}

When a patient complains of abnormal menstrual bleeding, it is first essential to obtain a thorough history regarding the changes in menstrual pattern, the frequency and duration of flow, the amount of blood loss, and any associated symptoms related to menstruation. ${ }^{9}$ In addition, a family history of bleeding problems, a sexual history, a medical and obstetrical history should be obtained. ${ }^{10}$ A complete physical examination should also be performed. These steps will help the provider to classify the type of abnormal bleeding and to ascertain whether the abnormality may be due to pregnancy-related bleeding, ovulatory bleeding or anovulatory bleeding. It can also allow the provider to determine whether an underlying systemic disorder or coagulation disorder is contributing to the abnormal uterine bleeding. Anovulatory menstrual cycles are more often seen in patients in the pubertal and perimenopausal years and are due to failure of the corpus luteum to sustain the developing endometrium. ${ }^{10}$ Anovulatory cycles can also be due to endocrine abnormalities. Ovulatory cycles that occur with abnormal uterine bleeding are more likely due to anatomic abnormalities. ${ }^{10}$ Further investigations of the patient's chief complaint can include laboratory studies and imaging modalities.

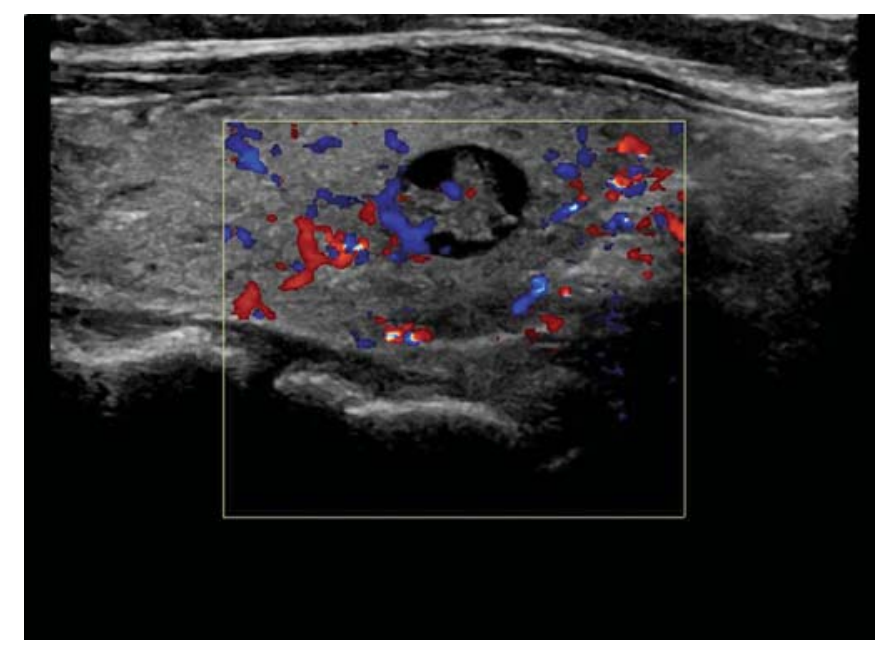

Fig. 8B: Color Doppler reveals increased vascularity at the periphery of a thyroid nodule 
The first step in management of abnormal uterine bleeding is to obtain a pregnancy test in all sexually active women of reproductive age. In addition, gonorrhea and chlamydia cultures should be studied. If the patient is not pregnant and cultures show no evidence of existing infection, management is continued to determine ovulatory $v s$ anovulatory cycles. If the patient is less than 20 years of age with heavy menstrual bleeding, coagulation studies should be performed, including the ristocetin cofactor assay for von Willebrand's disease. If the patient is over 35 years of age, an endometrial biopsy should be performed to rule out endometrial hyperplasia. To determine ovulatory vs anovulatory cycles, the provider can perform transvaginal ultrasound, hysteroscopy, or saline infusion ultrasonography to check for anatomic abnormalities. The provider can also check levels of thyroid hormone, estrogen, androgens, LH, FSH and prolactin. ${ }^{10}$

While performing pelvic ultrasound in nonpregnant patients with abnormal genital tract bleeding, always look for an enlarged uterus, which may indicate the presence of uterine fibroids. Carefully evaluate and describe the myometrium. ${ }^{11-13}$ If the myometrium is heterogeneous, assess and describe focal myometrial nodules, typical of uterine fibroids or leiomyoma. Each lesion should be measured in 2 to 3 diameters. Make sure that in your report you define the uterine mass location (subserosal/intramural/ submucosal) and specify the uterine mass echogenicity. Always define the uterine mass borders (well defined vs ill defined). Well-defined lesions are more likely to be uterine fibroids, while ill-defined lesions with endometrial and/or myometrial cysts are suggestive of adenomyosis. While assessing uterine adenomyosis specify the dimension and location of the cysts (within the inner, medial or deep myometrial layer), and evaluate the regularity of the endometrial-subendometrial layer. This is helpful in differentiating between superficial and deep adenomyosis.
In every patient presenting with abnormal uterine bleeding, assess the endometrial thickness (double layer in a long axis), and echogenicity (triple layer vs homogeneously hyperechogenic). Always look for focal abnormalities, suggestive of endometrial polyps. If you are not sure about the finding, perform saline infusion sonography (SIS) or hysterosonography. ${ }^{14}$ Describe whether there is evidence of intracavitary fluid.

The endometrial appearance has to be correlated with the ovarian findings (triple line endometrial appearance is typical for the follicular phase of the menstrual cycle, while homogeneously hyperechogenic endometrium is visualized in the luteal phase of the menstrual cycle). ${ }^{11-13}$

Adnexal evaluation includes precise ovarian measurements (3 diameters for each ovary and measurement of the ovarian volume using either vocal assessment or a formula for an ellipsoid, D $1 \times \mathrm{D} 2 \times \mathrm{D} 3 \times 0.523$ ). Ovarian volume exceeding 10 to $12 \mathrm{ml}$ with more than 10 follicles in ovarian long axis, measuring from 2 to $8 \mathrm{~mm}$ is suggestive of polycystic ovaries. ${ }^{11-13}$ Document every intraovarian or adnexal lesion and describe its sonographic characteristics. In patients with dysfunctional bleeding, look for the presence of an unruptured follicle and/or a simple ovarian cyst. Always report on the presence or absence of free fluid in the posterior cul-de-sac.

Table 1 reviews imaging methods used for the evaluation of patients presenting with abnormal genital tract bleeding. Although imaging methods can not replace definitive histologic diagnosis and tissue sampling, they are the most appropriate step in evaluating patients with premenopausal abnormal vaginal bleeding. ${ }^{14,15}$ Imaging plays an irreplaceable role in screening, characterization of structural abnormalities, and directing appropriate patient care, often preventing more invasive diagnostic procedures. ${ }^{15}$

Treatment of abnormal uterine bleeding usually begins with medical therapy to alleviate symptoms and control ovulation. Generally, oral contraceptive pills and

Table 1: Role of imaging in the evaluation of nonpregnant reproductive age patients with abnormal genital tract bleeding

\begin{tabular}{|c|c|}
\hline Imaging method & Comments \\
\hline Transabdominal ultrasound & $\begin{array}{l}\text { - Adjunct to transvaginal ultrasound } \\
\text { - Poor penetration due to uterine fibroids and adenomyosis }\end{array}$ \\
\hline Transvaginal ultrasound & $\begin{array}{l}\text { - Method of choice for evaluating abnormal uterine bleeding } \\
\text { - Assessment of the uterine (endometrial and myometrial) and ovarian lesions }\end{array}$ \\
\hline Color Doppler ultrasound & $\begin{array}{l}\text { - Assessment of uterine and ovarian vascularization } \\
\text { - May be of added value in characterization of the uterine and/or ovarian masses }\end{array}$ \\
\hline Saline infusion sonography & $\begin{array}{l}\text { - Identification of the intracavitary lesions } \\
\text { - Better delineation of the endometrium when it cannot be well visualized by } \\
\text { transvaginal ultrasound }\end{array}$ \\
\hline CT with contrast & - Not warranted for evaluation of the patient with abnormal vaginal bleeding \\
\hline MRI without and with contrast & $\begin{array}{l}\text { - Problem solving tool and adjunct to transvaginal ultrasound } \\
\text { - Useful when saline infusion sonography cannot be performed for technical reasons } \\
\text { - Useful for endometrial carcinoma staging }\end{array}$ \\
\hline
\end{tabular}


nonsteroidal anti-inflammatory agents are used. Surgical intervention is only considered when medical therapy fails. Therapy is tailored to the individual patient and her underlying pathology, as well as her concerns and contraindications. $^{10}$

\section{REFERENCES}

1. Rahn D, et al. Systematic review highlights difficulty interpreting diverse clinical outcomes in abnormal uterine bleeding trials. J Clin Epidemiol March 2011;64(3):293-300.

2. Liu Z, et al. A systematic review evaluating health-related quality of life, work impairment, and health-care costs and utilization in abnormal uterine bleeding. Value Health May-June 2007; 10(3):183-94.

3. Cote I, Jacobs P, Cumming D. Work loss associated with increased menstrual loss in the United States. Obstet Gynecol 2002;100:683-87.

4. Shwayder JM. Pathophysiology of abnormal uterine bleeding. Obstet Gynecol Clin North Am 2000;27:219-34.

5. Ehrmann D. Polycystic ovary syndrome. N Engl J Med 2005; 352:1223-36.

6. Peipert J. Genital chlamydial infections. N Engl J Med 2003; 349:2424-30.

7. Ginsburg D, Wagner D. Structure, biology and genetics of von Willebrand factor. In Hoffman R (Ed): Hematology: Basic principles and practice, (3rd ed). New York: Churchill Livingstone 2000:1937-45.

8. Schorge JO, et al. Abnormal uterine bleeding. Williams Gynecology. McGraw Hill: New York 2008.

9. Lobo RA. Abnormal uterine bleeding: Ovulatory and anovulatory dysfunctional uterine bleeding, management of acute and chronic excessive bleeding. In: Katz VL, Lentz GM, Lobo RA, Gershenson DM (Eds). Comprehensive Gynecology. (5th ed). Philadelphia, Pa: Mosby Elsevier 2007;37:915-31.

10. Bradley L. Investigation of abnormal uterine bleeding in premenopausal women. Hysteroscopy: Office evaluation and management of the uterine cavity (1st ed). Philadelphia: Mosby, Inc, 2009;10:95-114.
11. Kupesic S, Plavsic BM. Sonography of uterine leiomyomata. In: Brosens I (Ed): Uterine fibroids: Pathogenesis and management. London, New York: Taylor \& Francis 2006: 139-51.

12. Kupesic Plavsic S, Kurjak A, Baston K. Color Doppler and threedimensional ultrasound of the uterine lesions. In Kupesic Plavsic (Ed). Color Doppler, 3D and 4D ultrasound in obstetrics, gynecology and infertility. London, New Delhi, Panama City, St Louis, Jaypee Publishers 2011;22-32.

13. Kupesic Plavsic S. Pelvic ultrasound pearls. In: Kupesic Plavsic S. (Ed). Color Doppler, 3D and 4D ultrasound in obstetrics, gynecology and infertility. London, New Delhi, Panama City, St Louis, Jaypee Publishers 2011;256-348.

14. Kupesic Plavsic S, Plavsic BM. 2D and 3D saline infusion sonography and hystero-contrast-salpiongography. In Kurjak A, Chervenak F (Eds). Donald School Textbook of Ultrasound in Obstetrics and Gynecology. Jaypee Brothers Medical Publishers LTD; London, New Delhi, Panama City, St Louis 2011; 900-15.

15. Abnormal vaginal bleeding. ACR appropriateness criteria (last review date 2010). http://www.acr.org/SecondaryMainMenu Categories/quality_safety/app_criteria/pdf/ExpertPanelon WomensImaging/AbnormalVaginal BleedingDoc1.aspx

\section{ABOUT THE AUTHORS}

\section{Bailey Wilson}

Medical Student, Department of Obstetrics and Gynecology, Paul L Foster School of Medicine, Texas Tech University Health Sciences Center, El Paso, Texas, USA

\section{Sanja Kupesic Plavsic (Corresponding Author)}

Professor, Department of Obstetrics and Gynecology, Paul L Foster School of Medicine, Texas Tech University, 4800 Alameda Ave, El Paso, TX 79905, USA, Phone: +1 (915)783-1700, e-mail: sanja. kupesic@ttuhsc.edu

\section{QUESTIONS}

1. A 26-year-old obese female with irregular menses and hirsute features comes to your clinic. You suspect that she has PCOS. What laboratory abnormalities would you expect in this patient?
A. Decreased luteinizing hormone levels
B. Increased follicle stimulating hormone levels
C. Decreased prolactin level
D. Increased free testosterone

2. Which of the following is not a possible sequelae of chronic cervicitis?
A. Pelvic inflammatory disease
B. Fitz-Hugh-Curtis syndrome
C. Painless chancre
D. Infertility

3. A patient presents to your office with complaints of menorrhagia and dysmenorrhea for the past 6 months. Based on the patient's history and physical examination, you suspect an endometrial polyp. Which test or procedure would be most helpful in confirming this diagnosis?
A. CT of the pelvis
B. Saline infusion sonography 
C. Endometrial biopsy

D. Follicle stimulating hormone level

4. A 32-year-old nulliparous female complains of menometrorrhagia. One month ago she had a D and C, which has failed to resolve her symptoms. Pelvic ultrasound reveals a thickened endometrium and a simple left ovarian cyst measuring $4 \times 4 \mathrm{~cm}$. What is the most appropriate next step?
A. Perform hysterectomy
B. Perform hysteroscopy
C. Treat with birth control pills
D. Perform endometrial ablation

5. Which of the following is the best first-line treatment for a patient presenting with dysfunctional uterine bleeding?
A. Endometrial ablation
B. Hysterectomy
C. Oral contraceptive pills
D. Clomiphene citrate

6. In a patient with an ovarian cyst that has not resolved in the past $\mathbf{3}$ months, what would be the next step in care?
A. Oral contraceptives
B. Observation
C. Medroxyprogesterone
D. Laparoscopy

7. Which of the following is the most common presentation for a woman with a uterine leiomyoma?
A. Asymptomatic
B. Infertility
C. Vaginal bleeding
D. Pelvic pressure

8. Depict the typical pelvic ultrasound finding of a patient with intramural uterine fibroid
A. Uterine enlargement and irregular uterine contours
B. Heterogeneous myometrium
C. Well-delineate intramural nodule
D. All of the above

9. In a young female with PCOS and patent fallopian tubes, which of the following is the preferred initial method of ovulation induction?
A. GnRH analog
B. Clomiphene citrate
C. Gonadotropins
D. Oral contraceptive pills

10. Which of the following is the most likely cause of abnormal genital bleeding in a 13-year-old girl?
A. Uterine cancer
B. Ectopic pregnancy
C. Threatened abortion
D. Anovulation

\section{CORRECT ANSWERS}

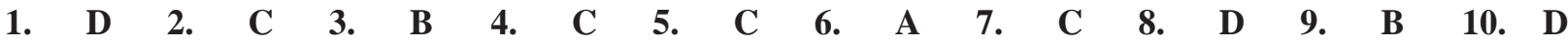

